\section{THE LIFE OF A STAR.}

\section{A LetTer from Prof. Perry to Sir Norman} LOCKYER.

YOU have asked me to examine certain publications on this subject, and to give you my views on the value of such speculations as have been made by mathematical physicists.

Mr. T. J. J. See (The Astronomical Journal, Boston, February 6, 1899) states as "one of the most fundamental of all the laws of nature "that gaseous masses follow the law

$$
t=\frac{\mathrm{K}}{\mathrm{R}}
$$

where $\mathrm{K}$ is a constant for all stars of whatever mass or of whatever kind of gaseous stuff, $\mathrm{R}$ is the radius and $t$ is the temperature. Now we have all sorts of temperatures in a star; but whether Mr. See takes average temperature or the temperature of some layer at a definite depth below the surface, he is certainly wrong. Mr. Homer Lane does not express the general results which I shall give presently, nor does Lord Kelvin give them in my form (although he does give them); but from either of these classical papers Mr. See might have inferred them, and seen that his own statement was wrong. Mr. See's arguments are really metaphysical. For example, at the very beginning of the proof of his proposition he speaks of the gravitational pressure at the surface of a star, whereas in physics we do not admit that there can be such a pressure in the absence of outside matter. Thus it is impossible for a mathematical physicist to get to Mr. See's point of view.

Of A. Ritter's articles in Wiedemann's Annalen there is a good abstract in the Astrophysical Journal, Chicago, December 1898 . He assumes that the radiating layer on the outside of a star is of constant mass. He also assumes that the rate of radiation is proportional to the fourth power of the average temperature of this layer. $\mathrm{He}$ is dealing with temperatures which are so much greater than the temperatures with which we work in the laboratory, that such assumptions must be regarded as quite arbitrary.

$\mathrm{Mr}$. Homer Lane, in his classical paper on the theoretical temperature of the sun (American Journal of Sience and Arts, second series, vol. 1. p. 57, 1870), makes the assumption that Dulong and Petit's law of radiation is true for solar radiation, and he uses it to calculate the temperature of the radiating layer, which he finds to be $28,000^{\circ} \mathrm{F}$. That is, he uses an empirical law, obeyed possibly at laboratory temperatures in radiation from hot solids, to express the radiation at enormous temperatures from a hot layer of gas which has layers of gas. of all sorts of temperatures above and below it.

It seems to me that we know too little about the phenomenon of radiation from layers of gas with denser and hotter layers below and rarer and colder layers above to allow of any weight being placed upon these assumptions of Ritter or Homer Lane. In a star we have layers of fluid at all sorts of temperature and density. We have no laboratory knowledge of radiation that is applicable. We know very little about any star except our own sun. During Palæozoic time, many millions of years, there has been life on our earth. Prof. Newcomb is of opinion that the sun's heat received by the earth cannot have varied more than a very little during Palæozoic time. My results will enable us to see what this uniformitarian assumption leads to. It is my own belief (see NATURE, p. 582, April 1895) that there may have been many millions of years during which the sun may have been radiating at only one-third or one-tenth of its present rate. My formula will enable us to apply such assumptions as these, and see what they lead to. However different assumptions of this kind may appear to be, they all lead to results which only differ in degree, and not in kind. Assumptions like those of Homer Lane and Ritter may lead to results which are altogether wrong.

All this is speculation, but it is speculation on physical and mathematical lines where criticism is immediately applicable to one's logic and one's premises.

\section{Gaseous Stars.}

Homer Lane, Lord Kelvin, Ritter, and all people who have tried to make exact calculations, have assumed that the stuff of which a star is composed behaves as a perfect gas in a state of convective equilibrium. I also assume that this is the case. But if we apply our results to our own sun, we find that at its centre there is a density 33 , that is, 50 per cent. greater than the ordinary density of platinum. It seems to me that speculation on this basis of perfectly gaseous stuff ought to cease when the density of the gas at the centre of the star approaches $0^{\prime} \mathrm{I}$ or onetenth of the density of ordinary water in the laboratory.

Let $\rho$ be density, $t$ absolute temperature, $p$ pressure of the gas at the distance $r$ from the centre ; the gas is such that $\rho \sigma t=p, \sigma$ being a constant depending on the nature of the gas, and let $\gamma$ be the ratio of its specific heats. Let there be convective equilibrium, so that

or

$$
\begin{aligned}
& \rho t^{x /(\varepsilon-\gamma)}=c_{1} \text {, a constant ..... (I) } \\
& p c^{\gamma /(\mathrm{r}-\gamma)}=c_{3} \text {, a constant ...... (2) }
\end{aligned}
$$

Let $t_{0}$ and $\rho_{0}$ be values at the centre of the star.

If $m$ is the mass inside the radius $r$, then

$$
\frac{d p}{d r}=-a \frac{m}{r^{2}} \rho \ldots \ldots . . .(3)
$$

[I introduce the constant $a$ because $\frac{m \rho}{r^{2}}$ is the gravitational force with which a mass $m$ attracts a mass $\rho$ at the distance $r$. If we keep $a=\mathrm{I}$, all our forces will be in gravitational units. I prefer to have them in laboratory units. If we keep to C.G.S. units throughout, as one dyne is the weight of one gramme at the earth's surface $\div 98 \mathrm{I}$ and the weight of one gramme corresponds to $\frac{M_{1}}{R_{1}^{2}}$ gravitation units, where $M_{1}$ is the mass of the earth in grammes and $R_{1}$ is the radius of the earth in centimetres; one dyne corresponds to $\frac{\mathrm{M}_{1}}{98 \mathrm{I} \mathrm{R}_{1}{ }^{2}}$ gravitation units, so that

Also

$$
\left.a=\frac{98 \mathrm{I} \mathrm{R}_{1}^{2}}{\mathrm{M}_{1}}\right] \text {. }
$$

(3) is the same as

$$
m=4 \pi \int_{0}^{r} r^{2} \rho \cdot d r \ldots . . .(4)
$$

$$
r^{2} c_{1} \frac{\gamma}{\gamma-1} \frac{d t}{d r}=-a n \ldots \ldots . . . .
$$

From $(4)$,

$$
\frac{d m}{d r}=4 \pi r^{2} \rho=4 \pi r^{2} c_{1} \frac{r}{r-1}
$$

Hence, differentiating ( 5 ), we have

$$
\frac{d^{2} t}{d r^{2}}+\frac{2}{r} \frac{d t}{d r}+\frac{4 \pi a(\gamma-\mathrm{I}) \rho_{0} t I !(\gamma-\mathrm{x})}{\sigma \gamma}=0 \cdots \cdots
$$

Let us assume that $t=t_{0} \theta$, and that $r=b x$, choosing $b$ so that $x$ and $\theta$ shall not depend upon $t_{\theta}$ or $\rho_{0}$, and that the coefficient of the last term is $I$, thus we find

$$
\frac{d^{\prime 2} \theta}{d x^{2}}+\frac{2}{x} \frac{d \theta}{d x}+\theta^{1 /(\gamma-1)}=0 \ldots . . .(7)
$$

an equation which is true for any star the $\gamma$ for whose gaseous stuff is known.

$\theta$ which is $t / t_{0}$ may be expressed as a sum of powers of

NO. I $55^{\circ}$, VOL. 6O] 
$x$, and so tabulated. In the same way, $\phi$ or $f / \rho_{0}$ might be tabulated. Indeed $\phi^{\gamma-1}=\theta$. Again $\mu$ or $\int_{0}^{x} \phi x^{2} . d x$ may be tabulated. Mr. Lane has done this work for several values of $\gamma$. Solution by means of series of powers of $x$ can be relied upon only till $x=\mathrm{I}$. After that one must work indirectly. Lord Kelvin, in a paper published in the Philosophical Magazine, I887, vol. xxiii. p. 287 , gives numbers calculated by his assistant, Mr. Magnus Maclean, from which, with the help of Mr. J. Lister's or Mr. Homer Lane's values at $x=\mathrm{I}$, I could give a table like the following for the case of $\gamma=\mathrm{I}^{*} 4$. There are outside limits for $x$ and $\mu$, which Mr. Lane calls $x^{\prime}$ and $\mu^{\prime}$. Knowing the value of $\theta$ for $x=\mathrm{I}, \mathrm{I}$ find that Lord Kelvin's numbers give $x^{\prime}$ as $5^{\circ} 24$, and the corresponding $\mu^{\prime}$ as $2^{\circ} \mathrm{I} 65$, whereas Mr. Lane gives $x^{\prime}$ as 5.35, and $\mu^{\prime}$ as $2^{\prime} \cdot 188$. Mr. Lane does not publish the other values, and his curves are drawn to too small a scale for us to be able to make out tables of the values of $\theta$ or $\phi$. Lord Kelvin from $x=0$, and Mr. Lane for values beyond $x=\mathrm{I}$, obtained their results by methods such that errors may have increased as the work proceeded.

On the whole, I am disposed to take Lord Kelvin's numbers with an $x^{\prime}$, which is the mean of those just given, or $5^{\circ} 30$, and $\mu^{\prime}$ as $2^{\prime} 177$.

TABLE I. - For Gaseous Stuff whose Specific Heat Ratio is I 4.

\begin{tabular}{|c|c|c|c|c|c|c|}
\hline$x$ & & $\theta$ & & $\phi$ & & $\mu$ \\
\hline & $\ldots$ & I. & $\ldots$ & I 1000 & $\ldots$ & 0 \\
\hline 795 & $\ldots$ & 904 & $\ldots$ & 777 & $\ldots$ & $\cdot 136$ \\
\hline .883 & $\ldots$ & $\cdot 884$ & $\ldots$ & 734 & $\ldots$ & $\cdot 184$ \\
\hline •993 & $\ldots$ & $\cdot 857$ & $\ldots$ & .679 & $\ldots$ & $25^{2}$ \\
\hline $\mathrm{I} \cdot \mathrm{I} 4$ & $\ldots$ & 819 & $\ldots$ & 607 & $\ldots$ & 355 \\
\hline$I \cdot 33$ & $\ldots$ & 763 & $\ldots$ & 508 & $\ldots$ & .512 \\
\hline I. 59 & $\ldots$ & $68 \mathrm{I}$ & $\ldots$ & 385 & $\ldots$ & 758 \\
\hline I.99 & .. & 562 & $\ldots$ & $\cdot 237$ & $\ldots$ & $I^{\prime} \times 33$ \\
\hline 2.65 & $\ldots$ & 384 & $\ldots$ & .0916 & $\ldots$ & 1.666 \\
\hline 3.97 & $\ldots$ & I4 I & $\ldots$ & . OO74 & $\ldots$ & $2 \cdot 117$ \\
\hline $5 \cdot 30$ & $\ldots$ & 0 & $\ldots$ & 0 & $\ldots$ & $2 \cdot 177$ \\
\hline
\end{tabular}

We know now that for any star whose stuff behaves like a perfect gas

$$
\left.\begin{array}{l}
r=\mathrm{A}\left(\frac{t_{0}}{\rho_{0}}\right)^{1 / 2} x, m=\mathrm{B} t_{0}^{3 / 2} \rho_{0}^{-1 / 2} \mu \\
t=t_{0} \theta, \rho=\rho_{0} \phi
\end{array}\right\} . . .(8)
$$

Where

$$
\mathrm{A}=\sqrt{\frac{\sigma \gamma}{4 \pi a(\gamma-1)}}, \text { and } \mathrm{B}=4 \pi \mathrm{A}^{3} .
$$

we see that $A$ and $B, x^{\prime}$ and $\mu^{\prime}$ depend merely on the nature of the gas. We have

$$
\begin{aligned}
& \mathrm{R}=\mathrm{A}\left(\frac{t_{0}}{\rho_{0}}\right)^{\frac{1}{2}} x^{\prime} \ldots \ldots \ldots \\
& \mathrm{M}=\mathrm{B} t_{0}^{3 / 2} \rho_{0}^{-\frac{5}{5}} \mu^{\prime} \ldots \ldots \ldots
\end{aligned}
$$

if $R$ is the outside radius and $M$ is the whole mass.

We may choose values of $t_{0}$ and $\rho_{0}$, and calculate $\mathrm{R}$ and $\mathrm{M}$, or it is easy to see that if we know $\mathrm{R}$ and $\mathrm{M}$, we may calculate the internal density and temperature by

$$
\left.\begin{array}{l}
t_{0}=\frac{x^{\prime}}{4 \pi \overline{\mathrm{A}^{2} \mu^{\prime}}} \cdot \frac{\mathrm{M}}{\mathrm{K}} \\
\rho_{0}=\begin{array}{c}
x^{\prime 3} \\
4 \pi \mu^{\prime}
\end{array} \\
4 \pi \\
\mathrm{R}^{3}
\end{array}\right\} . . . . .
$$

It will be noticed that, $\sigma$ being proportional to the molecular volume (being sixteen times as great in hydrogen as in oxygen), $\rho_{0}$ is independent of $\sigma$, whereas $t_{0}$ is inversely proportional to $\sigma$. If we consider our own sun to be made of hydrogen, and if the laws of perfect gases could be applied as we have applied them, $t_{0}=3^{.25} \times{ }_{10} 0^{7}$ degrees centigrade, $\rho_{0}=33$, that is, 50 per cent. greater than the density of platinum (see how I blush). Whereas if it were made of oxygen, $\rho_{0}$ is the NO. I 550 , VOL. 6o] same as before, but $t_{0}$ is $2.03 \times 10^{\prime 1}$ degrees. It is sometimes good to employ, instead of $(8)$

$$
\left.\begin{array}{l}
r=\frac{\mathrm{R}}{x^{\prime}} x, m=\frac{\mathrm{M}}{\mu^{\prime}} \mu \\
t=\frac{\mathrm{M} x^{\prime}}{4 \pi \mathrm{A}^{2} \mathrm{R} \mu^{\prime}} \theta, \quad \rho=\frac{\mathrm{M} x^{3}}{4 \pi \mathrm{R}^{3} \mu^{\prime}}, \phi
\end{array}\right\} \ldots . .
$$

The above tables and these formulæe enable us to find the temperature and density at any point in any gaseous star of any mass, size and material (if $\gamma$ is $1 \cdot 4$ ). The curve connecting $\theta$ and $x$ is the $t, r$ curve for any star; the curve connecting $\phi$ and $x$ is the $\rho, r$ curve for any star; the scales of measurement are given in (I 2 ).

The intrinsic energy (not including any gravitational energy) of the whole mass being $h$, since the intrinsic energy of unit mass at temperature $t$ is $k t$, if $k$ is the specific heat (in ergs) at constant volume, and $t$ is $t_{0} \rho_{0}{ }^{\mathrm{I}}-\gamma \rho \gamma-\mathrm{r}$

$$
h=4 \pi k t_{0} \rho_{0}{ }^{\mathrm{I}} \quad \gamma \int_{0}^{\mathrm{R}} r^{2} \rho \cdot d r
$$

if $\mathrm{X}$ stands for

$$
\begin{array}{r}
h=4 \pi k \mathrm{~A}^{3} t_{0}^{5 / 2} \rho_{0}{ }^{-\frac{1}{2} \mathrm{X}} \\
\int_{0}^{x^{\prime}} x^{2} \theta \gamma /(\gamma-\mathrm{r}) d x
\end{array}
$$

a known number depending only on the value of $\gamma$. Hence

$$
\left.h=\frac{k \mathrm{X} x^{\prime}}{4 \pi \mathrm{A}^{2} \mu^{\prime 2}} \cdot \frac{\mathrm{M}^{2}}{\mathrm{R}} \cdot \cdot \cdot \cdot \cdot \text {. ( } \mathrm{I}_{3}\right)
$$

If $\mathrm{W}$ is the work done by gravitation in bringing all the stuff into its present position from an infinite distance,

where

$$
\mathrm{W}=a 4 \pi \int_{0}^{\mathrm{R}} \rho m r \cdot d r=a \mathrm{Y} \frac{x^{\prime}}{\mu^{\prime 2}} \cdot \frac{\mathrm{M}^{2}}{\mathrm{R}} \cdot . \cdot(\mathrm{I} 4)
$$

$$
\mathrm{Y}=\int_{0}^{x^{\prime}} x \mu \phi \cdot d x
$$

a known number depending only on the value of $\gamma$.

We can now speculate on these results. If the pieces of stuff which come together to form the nebula are not mere molecules, but of the size of meteors such as reach our earth, W will not be much less than what is here stated. Indeed, we may say that even when a star ceases to be gaseous, and throughout its whole history the value of $W$ is so nearly what is given in (14), that (14) may be used generally in such speculations as these.

A gaseous star doubles all its temperatures and its intrinsic heat energy when its radius is halved. We see that if all stars are of the same gaseous stuff, the ratio of $h$ to $\mathrm{W}$ is constant for all stars at all times. Let us put $\mathrm{W}=\alpha \stackrel{\mathrm{M}}{\mathrm{R}}, h=\beta \stackrel{\mathrm{M}}{\mathrm{R}}$.

As $\mathrm{W}=h+\mathrm{H}$ if $\mathrm{H}$ is the total energy lost by the star by radiation, then

$$
\mathrm{H}=(\alpha-\beta) \frac{\mathrm{M}^{2}}{\mathrm{R}}
$$

As part of this heat was lost by the stuff before it became a spherical gaseous star, we may take as the heat lost from time $T=0$ when the radius was $R_{0}$ to the present time $T$, when the radius is $R$

$$
(\alpha-\beta) \mathrm{M}^{2}\left(\frac{\mathrm{I}}{\mathrm{R}}-\frac{\mathrm{I}}{\mathrm{R}_{0}}\right) \text {. }
$$

In the mass $M$ there are surfaces whose areas are proportional to $\mathrm{R}^{2}$, and whose temperatures are proportional to $\frac{M}{R}$. I shall assume as quite reasonable, that

$$
\left.\begin{array}{c}
\text { Total radiation per } \\
\text { year from a star }
\end{array}\right\} \propto \text { areas } \times\left(\text { temperatures) }{ }^{n}\right.
$$

where $n$ is some constant. 
It may be worth while here to use with this the assumption that our sun, when gaseous, radiated heat of the same amount every year ; of course $\mathrm{H}$ of (I 5 ) or (I6) is then proportional to time. (15) is the age of the sun from some zero of time until it had the radius $\mathrm{R}$; (I6) is the time taken to contract from radius $R_{0}$ to radius $R$.

Using (I7),

$$
\text { Rate of total radiation } \propto R^{2}\left(\frac{M}{R}\right)^{n} \text {. }
$$

we see that $n$ must be 2 for our sun. In our state of ignorance of the phenomenon of radiation from a star it may be presumptuous in me to say that this would be a very reasonable $\dot{a}$ priori assumption. Namely that rate of radiation is proportional to surface and square of average temperature. Anyhow it makes the task of pursuing the uniformitarian assumption less thankless. ${ }^{I}$

For any star then the total radiation in unit time is proportional to $\mathrm{M}^{2}$, and hence the time taken by any gaseous star in contracting from radius $R_{0}$ to radius $R$ is

$$
\mathrm{T} \propto \frac{\mathrm{I}}{\mathrm{R}}-\frac{\mathrm{I}}{\mathrm{R}_{0}^{-}} \text {. . . . . . . }
$$

being the same for any star, whatever its mass may be. How it depends on the nature of its material we do not know, as we are basing these speculations on an assumption as to the sun's radiation. Or counting age from some period in the nebulous state, which it is not easy to define.

$$
\text { temperature of star } \propto \text { age } \times \text { mass . . }
$$

We see that stars get to have higher and higher tem-

1 If total radiation from a star is proportional to surfaces $\times$ the $n$th power of temperatures

$$
\frac{d \mathrm{H}}{d \mathrm{~T}} \propto \mathrm{M}^{n} \mathrm{R}^{2-n}
$$

but from (16),

$$
\frac{d \mathrm{H}}{d \mathrm{~T}} \propto-\frac{\mathrm{M}^{2}}{\mathrm{R}^{2}} \frac{d \mathrm{R}}{d \mathrm{I}}
$$

Putting these equal and integrating we find $T$ as the time since the star was of radius $R_{0}$

I. Thus if $n=\mathrm{I}$,

$$
\mathrm{T} \propto\left(\mathrm{R}_{0}^{n-3}-\mathrm{R}^{n-3}\right) \frac{\mathrm{M}^{2-n}}{n-3}
$$

$$
\mathrm{T} \propto \mathrm{M}\left(\frac{\mathrm{r}}{\mathrm{R}^{2}}-\frac{\mathrm{I}}{\left.\mathrm{R}_{0}\right)^{2}}\right)
$$

It follows from this assumption that the rate of increase of temperature per annum is proportional to Mass

II. If $n=2$ as above, Mass

$$
\mathrm{T} \propto \frac{\mathrm{I}}{\mathrm{R}}-\frac{\mathbf{I}}{\mathrm{R}_{0}} .
$$

It follows from this that the rate of increase of temperature per annum is constant and is proportional to the mass of the star.

III. If $n=3$

$$
\mathrm{T} \propto \frac{\mathrm{I}}{\mathrm{M}} \log \frac{\mathrm{R}_{0}}{\mathrm{R}} .
$$

It follows from this that the temperature increases with time by the com pound interest law; that is, the rate of increase of temperature per annum proportional to the mass $\times$ temperature.

$$
\text { IV. If } n=4 \text {, }
$$

$$
\mathrm{T} \propto \frac{\mathrm{I}}{\mathrm{M}^{2}}\left(\mathrm{R}_{0}-\mathrm{R}\right) .
$$

In this case the rate of increase of temperature per annum is proportional to the square of the temperature.

Suppose it to be assumed that the radiation is mainiy from an outer layer, that this layer increases in temperature from $t=0$ at its outer surface to $t=t_{1}$ at its inner surface, the depth or thickness of it is

$$
\mathrm{D} \propto \frac{\sigma \mathrm{R}^{3}}{\mathrm{M}} \text {. }
$$

Thus the thickness of the layer is greater with stuff like Hydrogen than with Oxygen. As we really know nothing about how the total radiation from such a layer depends upon the thickness, I cannot use this in my calculations. It is however worth noting that from equal surface areas of layers all with the same range of temperature but of different depths or thicknesses $\mathrm{D}$, the radiation per second $\propto\left(\frac{M}{\bar{D}}\right)^{n / 2}$.

Thus in the case above, in assuming $n=2$, we are really assuming that the radiation from unit area of layer is inversely proportional to its thickness. Suppose we speak of the depth $D^{\prime}$ below the surface to reach a layer of a particular density $\rho_{1}$ then

$$
D^{\prime} \propto \frac{R^{2 \frac{1}{2}}}{M^{\frac{1}{2}}}
$$

the depth being independent of whether the stuff is Oxygen or Hydrogen. peratures as they get older, until they cease to behave as gaseous bodies throughout. The temperature outside is o. The depth below the surface at which there exists a layer of a particular temperature, say $5000^{\circ}$ Cent. absolute, is proportional to $\mathrm{R}^{2} / \mathrm{M}$, or if our rule as to time is right, the depth is inversely as the mass of a star multiplied by the square of its age. In a very old, massive star the layer at $5000^{\circ}$ is very close to the outside.

It seems to me that this is an important thing. A young star, a truly gaseous star, has great depth of radiating layer. I mean it is probably only at great depths from the free surface that we find the layer from which a continuous spectrum comes. I take it that it is only during collision of molecules that a continuous spectrum is given out; in the free-path state of a molecule it radiates its own light only. Great density and high temperature conduce to the giving out of the continuous spectrum. In old stars, like our sun, the layer of stuff capable of giving out white light is comparatively near the surface of the star. I can imagine a comparatively young star long before its heat energy is a maximum, not radiating energy very fast, but rather giving out bright line spectra light from the greater part of its area; in fact from all but its central parts.

I am very ignorant of your subject, but I take it that any star gives out a continuous spectrum with lines. The continuous spectrum is strong, and the lines relatively dark, in old stars ; the continuous spectrum is weak, and the lines bright, in new stars. In both cases the continuous spectrum is most intense, and the lines least intense at the central parts of a star. If a star is very new, so that it is not all gas, it will probably not be spherical, and one may have spectra quite different in different places and at different times.

\section{Stars in General.}

I suppose that many people will think the above speculation to be fairly safe. It is correct on the assumptions. One may apply it to any star until the central density approaches $O .1$ or one-tenth of that of water or even more. In the case of our sun, the theory may have been applicable from the time when his radius was twenty times what it is now until it was five times what it is now. Near the surface I assume the density and temperature to be very small, and probably there is no substance that will behave as a perfect gas near the zero of temperature even if its density is also nearly zero. But as the mass of stuff in this condition is small, we may, I think, use our hypothesis. Besides, we are neglecting more important things; many possible conditions difficult to specify; heterogeneity; violent convective rushing of stuff like iron vapour to the places of low temperature where it may undergo sudden condensation and fall as iron hail over large regions; also, intense electrical actions are certainly taking place. All this may be said to be superficial, affecting only a small portion of the whole mass. On the whole, then, we may take our theory of gaseous stars to be applicable to some portion of the life of any star.

I am on much less safe ground when I try to trace the history of a star after its material ceases to behave as a perfect gas, and yet, as I take it, this is very much the longest part of its career. I may only vaguely speculate on its long or short life as a nebula; as a confused mass of streams of meteors in which every collision generates gaseous masses at all kinds of temperatures; its record is fairly clear from the time [if there ever is such a period in the truly gaseous state] when it assumes the spherical shape [in all cases I am neglecting rotation] and gets hotter and hotter and smaller and smaller. If the law of radiation is the same in any star as in our sun, and if we take one year's loss of heat energy by our sun as the unit of energy ; if our unit of mass is the mass of our sun and if the sun's present radius is our unit of length, I

No. I 550 , VOL. 6o] 
find ${ }^{2}$ [using Lord Kelvin's popular lecture figures for the present solar radiation] for any star,

$$
\begin{gathered}
\mathrm{W}=36 \times 10^{6} \frac{\mathrm{M}^{2}}{\mathrm{R}} \ldots \ldots . . . \\
h=32.4 \times 10^{6} \frac{\mathrm{M}^{2}}{\mathrm{R}} \ldots \ldots
\end{gathered}
$$

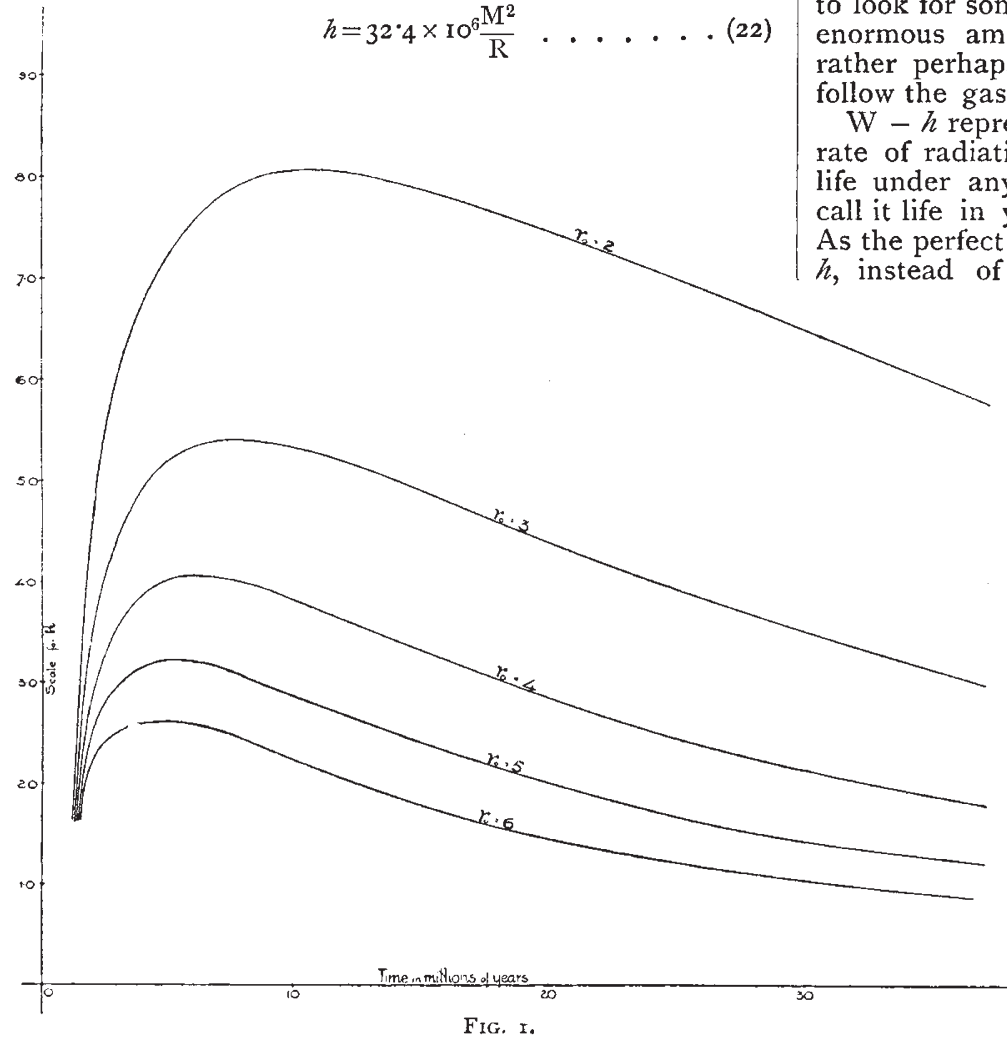

I must say that when Mr. Lister first worked out this value of $h$ for me I was greatly surprised, for it has been doubt (see also my final statement) that in a gaseous star the intrinsic or thermodynamic energy in the star is a very large fraction of the whole energy of the gravitating matter. Indeed it is so large that one is tempted to look for some greater original store to account for the enormous amount of radiation which takes place, or rather perhaps to assume that no radiating mass can follow the gaseous law.

resents life in years if we assume a uniform It has an obvious connection with nder any assumption that we may make. Let us life in years, and continue to consider our sun. the perfect gas law ceased more and more to be true, increasing steadily, reached a liniting value and then diminished again, so that eventually $h$ must become zero. In what state is our sun now? Is it still very much like a gas throughout, and getting hotter? It is too much to assume for stuff that would be 50 per cent. greater in density than platinum at the centre. In all probability the change from the law of (22) began before $R$ was 5 , was quite marked when $R$ was 4 , and $h$ reached its maximum value when $R$ was 4 or $3 \frac{1}{2}$ or 3 . It is quite certain that $h$ must reach a maximum value in any star, and afterwards diminish gradually, and the simplest mathematical formula expressing this fact may be used instead of (22) to give us useful suggestions in regard to the history of our sun. Such a simple formula is

$$
h=0.9 \mathrm{~W} /\left(\mathrm{I}+\frac{\mathrm{R}_{\theta}^{2}}{\mathrm{R}^{2}}\right) \ldots .
$$

If $\mathrm{R}$ is very great (23) is the same as (22). When $\mathrm{R}$ is $\mathrm{R}_{0}, h$ reaches a maximum value, and for smaller values of $\mathrm{R}, h$ diminishes. The following tables have been calculated, a different assumption being made for each. $W$ is calculated from $(2 I)$. $R_{0}$ is

\begin{tabular}{|c|c|c|c|c|c|c|c|c|c|c|c|c|c|c|c|c|c|c|c|c|}
\hline \multirow{2}{*}{$\begin{array}{c}\begin{array}{c}\text { Age of Star } \\
\text { in millions } \\
\text { of years. }\end{array} \\
T\end{array}$} & \multicolumn{4}{|c|}{$\mathrm{R}_{0}=6$} & \multicolumn{4}{|c|}{$\mathrm{R}_{0}=5$} & \multicolumn{4}{|c|}{$R_{0}=4$} & \multicolumn{4}{|c|}{$\mathrm{R}_{0}=3$} & \multicolumn{4}{|c|}{$\mathrm{R}_{0}=2$} \\
\hline & $\mathrm{w}$ & $h$ & $\mathrm{R}$ & $\mathrm{D}$ & $\mathrm{w}$ & $h$ & $\mathbf{R}$ & D & w & $h$ & $\mathrm{R}$ & D & w & $h$ & $\mathrm{R}$ & $D$ & W & $h$ & $\mathbf{R}$ & D \\
\hline $0^{\circ}$ & 3 'I 5 & 2.75 & $\mathrm{II}^{\circ} 42$ & $129^{\circ} 9$ & 2.5 이 & $2 \cdot 10$ & 14.39 & $208 \cdot 3$ & $2 \cdot 20$ & $\mathrm{I} \cdot 8 \mathrm{O}$ & $16 \cdot 34$ & $270^{\circ} 3$ & $2 \cdot 52 !$ & 2.12 & 14.29 & $204^{1} 1$ & 3.20 & 2.80 & 12.87 & $166 \%$ \\
\hline 0.8 & $2 \cdot 93$ & $2 \cdot 13$ & $12 \cdot 27$ & $149^{\circ} 3$ & $3 \cdot 28$ & 2.48 & 10.96 & 120.5 & 3.53 & $2 \cdot 73$ & 10.20 & $104 \cdot 2$ & $4 \cdot 12$ & $33^{2}$ & 8.802 & $76 \cdot 34$ & $5 \cdot 68$ & 4.48 & $6 \cdot 39$ & $40^{\circ} \mathrm{I}$ \\
\hline$I \cdot 5$ & 3.94 & 2.44 & $9 \cdot 229$ & 83.33 & 430 & $2 \cdot 80$ & 8.433 & $69^{\circ} 93$ & 4.82 & $3 \cdot 32$ & $7 \cdot 568$ & $55^{\circ} 87$ & $5 \cdot 65$ & $4=5$ & $6 \cdot 448$ & $40 \cdot 65$ & 7.01 & $5.5 \mathrm{I}$ & $5^{\circ} 173$ & $26 \cdot 39$ \\
\hline $2 \cdot 0$ & 4.52 & 2.52 & 8.038 & $63^{\circ} 69$ & 4.96 & 2.96 & 7.337 & 52.91 & $5 \cdot 57$ & 3.57 & 6.519 & $42^{\circ} .02$ & 6.50 & $450=$ & 5.573 & 30.77 & 8.01 & 6.01 & $4^{\circ} 5$ & $20 \cdot 2$ \\
\hline $3^{\circ} 0$ & 559 & 2.59 & 6.460 & $4 \mathrm{I} \cdot 49$ & $6 \cdot 14$ & $3 \cdot 14$ & 5.898 & 34.36 & 6.85 & 3.85 & $5 \cdot 268$ & $27 \cdot 62$ & 794 & 4.94 & 4454 & 19.69 & 9.78 & 6.78 & $3^{6} 699$ & 13.55 \\
\hline $4^{\circ} \mathrm{O}$ & 6.61 & $2.6 \mathrm{I}$ & 5.476 & $29 \cdot 67$ & $7 \cdot 23$ & $3 \cdot 23$ & $4^{\circ} 975$ & $24 \cdot 8 \mathrm{I}$ & 8.01 & $4.0 \mathrm{I}$ & 4.515 & $20 \cdot 20$ & 9.19 & $5 \times 19$ & 3.927 & $15 \cdot 38$ & $\mathrm{I} I \cdot 28$ & $7 \cdot 28$ & $3^{1} 198$ & IO' 1 \\
\hline $5^{\circ} 0$ & $7 \cdot 60$ & 2.60 & 4739 & 2247 & $8 \cdot 23$ & $3^{\circ} 23$ & $7 \cdot 367$ & $\left|19^{\circ} 16\right|$ & $9^{\circ} 06$ & 4.06 & 3.992 & $15.8 \mathrm{C}$ & $10^{\circ} 33$ & 5.33 & 3.508 & $12 \cdot 15$ & $12.61 \mid$ & $7 \cdot 61$ & 2.859 & 8.24 \\
\hline$\circ$ & 8.53 & $2 \cdot 53$ & 4219 & 17.79 & 9.14 & 3.14 & $3 \circ 937$ & I5.53 & 10.03 & $4^{\circ} 03$ & 3.584 & I 2.89 & I I 49 & 5.49 & $3 \cdot 137$ & $9^{\circ} 946$ & $13^{\circ} 83$ & $7 \cdot 83$ & $2 \cdot 605$ & $6 \cdot 83^{2}$ \\
\hline 8.0 & 10.33 & $2 \cdot 33$ & 3.484 & 12.15 & 10.54 & $2 \cdot 54$ & $3.4 \mathrm{I} 3$ & $\begin{array}{lll}1 & 1 & 67\end{array}$ & I I $9 I$ & $3.9 \mathrm{I}$ & 3.021 & 9.219 & 13.37 & $5 \cdot 37$ & 2.698 & 7246 & $|16.07|$ & 8.07 & $2 \cdot 244$ & $5^{\circ} .72$ \\
\hline I $20^{\circ} \mathrm{O}$ & 13.96 & $I .96$ & 2.577 & 6.667 & 14.59 & 2.59 & 2.463 & $6.116 \mid$ & 15.52 & 3.52 & $2 \cdot 320$ & & 17.08 & 508 & $2 \cdot 126$ & $4 \cdot 505$ & 20.04 & 8.04 & I.8OI & $3 \cdot 226$ \\
\hline I8.0 & 19.46 & $I \cdot 46$ & $1 \cdot 848$ & 3425 & 20.05 & 2.05 & I'792 & $3 \cdot 226$ & 20.96 & $2 \cdot 96$ & 1.715 & 2.950 & 22.48 & 4.48 & I 604 & 2.564 & $25^{\circ} 6 \mathrm{I}$ & $7^{6} 61$ & 1407 & I 976 \\
\hline $24^{\circ} 0$ & $25^{\prime 22}$ & $\mathrm{I} \cdot 22$ & 1.427 & $20^{\circ} 037$ & 25.68 & I.68 & $I^{*} 40 \mathrm{I}$ & I.96I I & $26 \cdot 44$ & $2 \cdot 44$ & $1 \cdot 362$ & I. 855 & 27.93 & 3.93 & $1 \cdot 291$ & $1 \cdot 667$ & $3 \mathrm{I}^{\circ} \mathrm{-04}$ & 7.04 & $\mathrm{I} \cdot \mathrm{I} 6 \mathrm{O}$ & $I \cdot 346$ \\
\hline $34^{\circ} \mathrm{I}$ & 34.98 & 0.88 & I 028 & $\mid \begin{array}{l}1.057 \\
\mid\end{array}$ & $35 \cdot 36$ & I.26 & I.O18 & 1.037 & 35.99 & $I .89$ & I & 1.000 & $37 \cdot 26$ & 3.16 & 9754 & 9346 & $40^{\circ} 03$ & 6.03 & 9024 & \\
\hline
\end{tabular}
the radius of our sun (as compared with its present

TABLE II. - Based on five different assumptions as to the time when our Sun was at its hottest. Also assuming Radiation at present rate.

generally thought that $h$ is always, not merely much less than W, but exceedingly less. But there can be no

${ }^{1}$ In C.G.S. units ( $\mathrm{I}_{3}$ ) and (14) give, if the stuff is like oxygen or hydrogen whose $\gamma$ is $x^{\circ} 4$, $h=6 \cdot 36 \times 10^{-8} \mathrm{M}^{2} / \mathrm{R}$
$\mathrm{W}=7.079 \times 10^{-8} \mathrm{M}^{2} / \mathrm{R}$

In obtaining these numbers $\mathrm{Mr}$. J. Lister took the values of $\theta$ deduced from Mr. Homer Lane's curves before we discovered Lord Kelvin's paper. It will be seen that he gets $h / \mathrm{W}=\cdot 9$. It is easy to show that this ratio must willy be 0.83 at het If our $\gamma$ is slightly different from ${ }^{\circ} 4$, we may have the above numbers. radius) when $h$ was a maximum. Thus the table headed $\mathrm{R}_{0}=4$ gives $\mathrm{W}$ and $h, \mathrm{~T}$ and $\mathrm{D}$ on the assumption that our sun reached its hottest condition when it was of 4 times its present radius. I take $\mathrm{W}-h$ and call it $\mathrm{T}$ the age or Time in years, but all these values of ' $T$ may be multiplied by some constant, $\mathrm{W}, h$ and $\mathrm{T}$ are given in millions.

$D$ is the depth (from surface) of a layer of stuff, say I0,000 ${ }^{\circ}$., taking the depth of such a layer at present as I.

Fig. I shows how the intrinsic heat energy of our sun NO. I 550 , voL. 6o] 
has varied with its age on the above assumptions, which are all uniformitarian. The curves and table will suit any star if the unit of energy employed is the heat radiated per year by the star. If a star is twice the mass of our sun, the unit of energy is four times as great as in the case of our sun. A curve connecting $R$ and time is

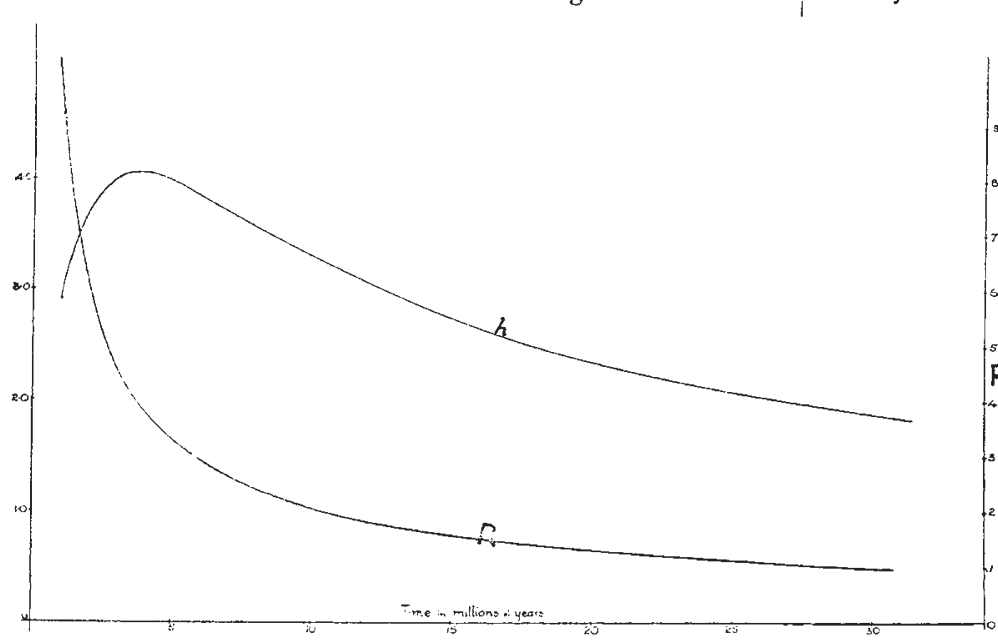

FIG. 2.

the same for all stars, and in the table the sun's present radius is the unit for $R$.

I take the critical size or size of maximum $h$ in a star to depend upon $\rho_{0}$ the central density; if then the critical radius of our sun was 4 (or 4 times its present radius), the critical radius of a star whose mass is $M$ times that of our sun was $4 \sqrt[3]{\mathrm{M}}$.

Non-Uniformitarian Assumptions.

The numbers in Table II. enable us to find what any assumption as to rate of radiation leads to. Thus, instead of assuming a constant amount of radiation every year, let us assume in the case of our sun that the rate of radiation at any time was always proportional to $h$. Let us take the supposition that $h$ was greatest for our sun when $R$ was four times its present value. Then as $T$ in the table is no longer to be called time, as it is really $\mathrm{W}-h$; let $t$ be time ; $c$ being some constant.

Hence

$$
\delta \mathrm{T}=c h . \delta t \text {. }
$$

$$
t=\int \frac{d \mathrm{~T}}{c h} \text {. }
$$

It is quite easy to plot the curve whose ordinate is $\frac{I}{c h}$ and whose abscissa is $T$ of the table; in this way using a value of $c$, which is suitable, $I$ find

\begin{tabular}{|c|c|c|c|c|c|c|c|}
\hline $\mathrm{W}-h$ & & $\frac{I}{h}$ & & $\begin{array}{l}\text { Age in } \\
\text { millions of } \\
\text { years. } \\
t .\end{array}$ & & R. & \\
\hline I & $\ldots$ & $\cdot 342$ & $\ldots$ & I'O & $\ldots$ & $9 \cdot 18$ & $\ldots$ \\
\hline 2 & $\ldots$ & .28 & $\ldots$ & I.78 & $\ldots$ & $6 \cdot 5$ & $\ldots$ \\
\hline 3 & .,. & .260 & $\ldots$ & I. 45 & $\ldots$ & $5 \cdot 27$ & $\ldots$ \\
\hline 4 & $\ldots$ & 250 & $\ldots$ & 20 & $\ldots$ & $45^{\circ}$ &.. \\
\hline 5 & $\ldots$ & $\cdot 24$ & $\ldots$ & $2 \cdot 71$ & $\ldots$ & 3. & $\ldots$ \\
\hline 6 & $\ldots$ & $\cdot 248$ & $\ldots$ & 333 & $\ldots$ & $3 \cdot 5$ & $\ldots$ \\
\hline 8 & $\ldots$ & $\cdot 256$ & $\ldots$ & 45 & $\ldots$ & 3.02 & $\ldots$ \\
\hline I 2 & $\ldots$ & $\cdot 284$ & $\cdots$ & $7 \cdot 2$ & $\ldots$ & $2 \cdot 32$ & $\ldots$ \\
\hline 18 & $\ldots$ & $\cdot 33^{8}$ & $\ldots$ & II ${ }^{\circ} 92$ & $\ldots$ & I $7 \mathrm{I}$ & $\ldots$ \\
\hline 24 & $\ldots$ & 41 & $\ldots$ & I $7^{\circ}$ & $\ldots$ & I'36 & $\ldots$ \\
\hline 34 & $\ldots$ & $\cdot 528$ & $\ldots$ & 29.26 & $\ldots$ & $1 . \infty$ & $\cdots$ \\
\hline
\end{tabular}

TABLE III.-Rate of Radiation Proportional to h.

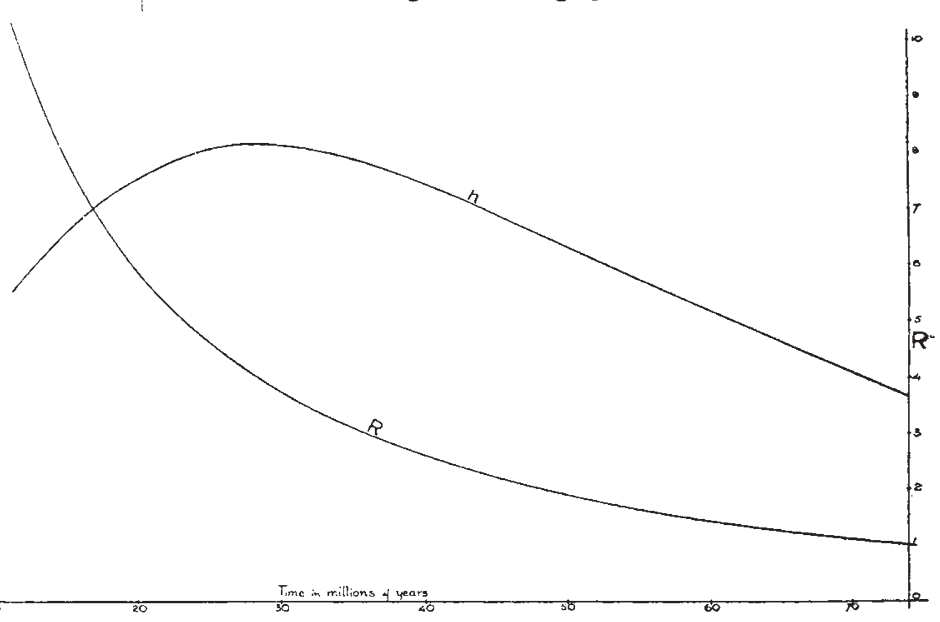

FIG. 3.

On no one of the above assumptions can I see that it is possible to give even a probable limit to the future life of our sun as a light-giving body.

\section{Energy in a Spherical Mass of Gas.}

I end this long letter with a very curious statement concerning gaseous masses in space, and I am sorry that my own proper work is demanding so much of my attention that I must leave the following very definite statement without applying it, as I see that it may be applied, to the study of the physical properties of many gases. We have seen that, under convective equilibrium, there is an outside radius beyond which there is no stuff

NO. I 55 O, VOL. 60$]$ 
existing. The following statement does not assume convective equilibrium ; an outside radius, $R$, is assumed to exist.

Let temperature, pressure, \&c., be functions of $r$. If $m$ is the total mass bounded by the spherical surface of radius $r$,

$$
\begin{gathered}
\frac{d p}{d r}=-a \frac{m}{r^{2}} \rho \ldots \ldots \\
m=4 \pi \int_{0}^{r} r^{2} \rho \text { dr . . . . . . . . }
\end{gathered}
$$

the stuff being a perfect gas,

$$
\rho \sigma t=p . \ldots . . .
$$

If $k$ is the specific heat (in ergs) at constant volume, the total intrinsic energy of the mass is

$$
\begin{aligned}
& h=4 \pi k \int_{0}^{\mathrm{R}} r^{2} \rho t \cdot d r, \\
& h=\frac{4 \pi k}{\sigma} \int_{0}^{\mathrm{R}} p r^{2} \cdot d r
\end{aligned}
$$

The work that would have to be done in taking successive layers to an infinite distance is

$$
\mathrm{W}=+a \int_{0}^{\mathrm{R}} \frac{m}{r} 4 \pi r^{2} \cdot d r \cdot \rho=+4 \pi a \int_{0}^{\mathrm{R}} \rho m r \cdot d r
$$

Now (24) is

$$
\rho a m=-r^{2} \frac{d p}{d r}
$$

so that

$$
\mathrm{W}=-4 \pi \int_{0}^{\mathrm{R}} r^{3} \frac{d p}{d r} d r=-4 \pi \int_{p_{0}}^{0} r^{3} d p \ldots
$$

Now in $(27)$

$$
\int_{0}^{\mathrm{R}} p r^{2} \cdot d r=\left[\frac{1}{3} p r^{3}\right]_{0}^{\mathrm{R}}-\int_{p_{0}}^{0} \frac{r^{3}}{3} d p
$$

The bracket term is o, as $r$ is o at the centre, and $p=0$ at the surface. Hence 27 is

$$
h=-\frac{4 \pi k}{\sigma} \int_{p_{0}}^{0} \frac{r^{3}}{3} d p
$$

Dividing by (29) we have

$$
\frac{h}{\mathrm{~W}}=\frac{k}{3^{\sigma}}
$$

Now in gases, if $\mathrm{K}$ is specific heat at constant pressure $\sigma=\mathrm{K}-k$ so that

If

$$
\begin{gathered}
\frac{h}{\mathrm{~W}}=\frac{k}{3(\mathrm{~K}-k)}=\frac{\mathrm{I}}{3(\gamma-\mathrm{I})} \\
\gamma=\mathrm{I} \frac{1}{3}, h=\mathrm{W} \\
\gamma=\mathrm{I} \cdot 4, h=\cdot 833 \mathrm{~W}
\end{gathered}
$$

If

Here are very definite astonishing statements !

I must confess that I do not understand how if $\gamma=I \frac{1}{3}$ we can have $h=\mathrm{W}$. It seems to mean that if a mass of this kind of gas gravitates by itself from an infinite distance it retains all its energy. But such gas must surely be imagined to be radiating heat, as it is not at zero temperature. Where can it get such heat ? I come to the conclusion that there must be atomic energy available somehow in it, even when we imagine the molecules at an infinite distance from one another, or else there is no such gas possible. I say that no substance for which $\gamma=\mathrm{I} \frac{1}{3}$ can behave as a perfect gas.

You will notice that we do not need to imagine our stuff in a state of infinite diffusion. If a gaseous star changes its size or the arrangement of its stuff, the gravitational work done is exactly equal to the additional intrinsic heat energy in the star if $\gamma$ is $I \frac{1}{3}$. The paradox is greater if we think of coloured diatomic gases such as chlorine, NO. I 550 , VOL. 60] which have values of $\gamma$ less than $I \frac{1}{3}$. We must either assume that there is more energy available than mere gravitational energy, or else that such substances cannot really behave as perfect gases. [It is to be remembered that by a perfect gas I do not merely mean that $p / t \rho$ is constant, but that $k$, the specific heat at constant volume is constant, a statement which does not follow from the first.] It is some time since I have come across a statement which looks better worth study than this one.

WILLIAM HENRY FLOWER, K.C.B., F.R.C.S., LL.D., D.C.L., SC.D., F.R.S., F.Z.S., F.L.S.

THE distinguished naturalist whose death has recently occurred was the second son of the late Mr. Edward Fordham Flower, the founder of the well-known brewery at Stratford-on-Avon, and dear to all lovers of animals on account of his crusade against the bearingrein. Sir William Flower was born in November $183 \mathrm{I}$. $\mathrm{He}$ was educated at private schools and at University Collese, London, where he took Sharpey's gold medal in Physiology, and Grant's silver medal in Zoology. He became M.B. of the University of London in $185 \mathrm{I}$, and joined the Medica! Department of the Army in 1854 , serving in the Crimea, where his health broke down. On his return to England he became Demonstrator of Anatomy at the Middlesex Hospital, and Curator of the Museum, intending to practise as a surgeon. Here he published his first work, "Diagrams of the Nerves of the Human Body" and also wrote in Holmes" "System of Surgery." on "Injuries of the Upper Extremities."

In 1861 , at the age of thirty, he was appointed to succeed Queckett as Curator of the Hunterian Museum at the College of Surgeons, and later became Hunterian Professor. Thenceforward he abandoned professional work for purely scientific pursuits. Twenty years later, when he received the Royal medal of the Royal Society, the President stated with justice that "it is very largeiy due to his incessant and well-directed labours that the museum of the Royal College of Surgeons at present contains the most complete, the best ordered, and the most accessible collection of materials for the study of vertebrate structures extant."

'Two years later (in 1884 ), on the resignation of Sir Richard Owen, Prof. Flower was appointed Director of the new Natural History Museum in the Cromwell Road, where he was incessantly occupied with the arrangement and development of the collections until failing health necessitated his resignation, which took effect in October 1898 . Unhappily he did not long enjoy the rest and leisure which he had so well earned by a life of unusual industry and devotion to public work.

His services in the cause of knowledge were recognised by many honorary degrees from Universities, and by his election as a Correspondent of the Institute of France. He was made C.B. in 1887 , and K.C.B. in 1892 , and was President in 1889 of the British Association for the Advancement of Science.

The mere enumeration of the incidents in a man's life does not tell very much about the nature and value of his work. Sir William Flower's chief work was in two directions: firstly, as a director and original artist in museum management; secondly, as an investigator and discoverer in the comparative anatomy of the Mammalia. Besides these two chief lines of work, there were others to which he gave time and care. He was not unheedful of the popular demand for instruction and guidance by lectures. He frequently appeared at the Royal Institution and the London Institution, and always had a weighty and well-considered discourse to deliver. The most original and, from a social point of view, the most important of these was one on "Fashion in Deformity," in which he gave very strong support to those who dis- 